\title{
Satiety testing in diabetic gastroparesis: Effects of insulin pump therapy with continuous glucose monitoring on upper gastrointestinal symptoms and gastric myoelectrical activity
}

\author{
Kenneth L. Koch ${ }^{1}$ ｜ William L. Hasler ${ }^{2}$ iD ｜ Mark Van Natta ${ }^{3}$ ～Jorge Calles-Escandon ${ }^{4}$ | \\ Madhusudan Grover ${ }^{5}$ (D) | Pankaj J. Pasricha ${ }^{6}$ | William J. Snape ${ }^{7}$ | Henry P. Parkman ${ }^{8}$ (D) | \\ Thomas L. Abell $^{9}$ (D) | Richard W. McCallum ${ }^{10}$ (i) | Linda A. Nguyen ${ }^{11}$ (D) | \\ Irene Sarosiek $^{10}$ | Gianrico Farrugia ${ }^{12}$ | James Tonascia ${ }^{3}$ | Linda Lee ${ }^{3}$ | Laura Miriel ${ }^{3}$ | \\ Frank Hamilton $^{13}$ | for the NIDDK Gastroparesis Clinical Research Consortium \\ ${ }^{1}$ Section on Gastroenterology, Wake Forest University, Winston-Salem, North Carolina \\ ${ }^{2}$ Division of Gastroenterology, University of Michigan, Ann Arbor, Michigan \\ ${ }^{3}$ Johns Hopkins University Bloomberg School of Public Health, Baltimore, Maryland \\ ${ }^{4}$ Endocrinology Section, MetroHealth Medical Center, Cleveland, Ohio \\ ${ }^{5}$ May Clinic, Rochester, Minnesota \\ ${ }^{6}$ Center for Neurogastroenterology, Johns Hopkins Bayview Medical Center, Baltimore, Maryland \\ ${ }^{7}$ California Pacific Medical Center, San Francisco, California \\ ${ }^{8}$ Section of Gastroenterology, Temple University, Philadelphia, Pennsylvania \\ ${ }^{9}$ Digestive and Liver Health, University of Louisville, Louisville, Kentucky \\ ${ }^{10}$ Division of Gastroenterology, Texas Tech University, El Paso, Texas \\ ${ }^{11}$ Division of Gastroenterology, Stanford University, Palo Alto, California \\ ${ }^{12}$ Division of Gastroenterology and Hepatology, Mayo Clinic, Rochester, Minnesota \\ ${ }^{13}$ National Institute of Diabetes and Digestive and Kidney Diseases, Bethesda, Maryland
}

\section{Correspondence}

Kenneth L. Koch, Section on

Gastroenterology, Wake Forest School of Medicine, Medical Center Boulevard, Winston-Salem, NC 27157.

Email:kkoch@wakehealth.edu

Funding information

The Gastroparesis Consortium (GpCRC) is supported by the National Institute of Diabetes and Digestive and Kidney Diseases (NIDDK) (grants U01DK112193, U01DK112194, U01DK073983, U01DK073975, U01DK074035, U01DK074007, U01DK073985, U01DK073974, U01DK074008) and the National Center for Advancing Translational Sciences (NCATS) (grants UL1TR000424, UL1TR000093, UL1TR000433, UL1TR000135).

\begin{abstract}
Background: Symptoms induced by caloric or non-caloric satiety test meals and gastric myoelectrical activity (GMA) have not been studied in patients with diabetic gastroparesis (DGP) before and after intense glucose management.

Aims: We determined the effects of continuous subcutaneous insulin infusion (CSII) with continuous glucose monitoring (CGM) on Gl symptoms, volume consumed, and GMA induced by the caloric meal satiety test (CMST) and water load satiety test (WLST) in DGP.

Methods: Forty-five patients with DGP underwent CMST and WLST at baseline and 24 weeks after CSII with CGM. Subjects ingested the test meals until they were completely full. Visual analog scales were used to quantify pre- and postmeal symptoms,
\end{abstract}


and GMA was recorded with cutaneous electrodes and analyzed visually and by computer.

Key Results: At baseline and 24-week visits, nausea, bloating, abdominal discomfort, and fullness were immediately increased after CMST and WLST (Ps < 0.01). The meal volumes ingested were significantly less than normal controls at both visits in almost one-third of the subjects. After the CMST, the percentage 3 cycle per minute GMA increased and bradygastria decreased compared with WLST (Ps < 0.05). After treatment for 24 weeks meal volumes ingested, postmeal symptoms and GMA were no different than baseline.

Conclusions and inferences: (a) Satiety test meals elicited symptoms of nausea, bloating, and abdominal discomfort; (b) CMST stimulated more symptoms and changes in GMA than WLST; and (c) CSII with CGM for 24 weeks did not improve symptoms, volumes ingested, or GMA elicited by the two satiety test meals in these patients with diabetic GP. Satiety tests in diabetic gastropresis are useful to study acute postprandial symptoms and GMA, but these measures were not improved by intensive insulin therapy.

\section{KEYWORDS}

caloric and non-caloric test meals, diabetic gastroparesis, gastric dysrhythmias, gastric myoelectrical activity, gastroparesis cardinal symptom index, patient assessment of upper GI symptoms

\section{1 | INTRODUCTION}

Early satiety, fullness, abdominal discomfort, bloating, and nausea and vomiting are symptoms associated with gastroparesis that usually increase after ingestion of meals. ${ }^{1}$ The rate of gastric emptying of solid test meals, which is used to define gastroparesis, is poorly related to these postprandial symptoms. ${ }^{2,3}$ Thus, the origin of postprandial symptoms in gastroparesis remains unclear. Provocative test meals have not been studied to assess immediate postprandial symptoms and gastric myoelectrical activity (GMA) in patients with diabetic gastroparesis.

On the other hand, test meals have been frequently used to study postprandial symptoms in patients with postprandial distress syndrome or dysmotility-like functional dyspepsia. These patients ingested lower volumes of caloric meals or non-caloric water loads compared with healthy control subjects and reported upper gastrointestinal symptoms similar to those reported by patients with gastroparesis. ${ }^{4-7}$ Patients with functional dyspepsia (dysmotility subtype) ingested smaller volumes of water and developed symptoms and a variety of gastric dysrhythmias compared with healthy subjects. ${ }^{7}$ In subjects with gastroparesis, lower volumes of water ingested were associated with increased early satiety, postprandial fullness, and severity of delayed emptying. ${ }^{8}$ Thus, disorders of gastric capacity (accommodation or hypersensitivity) and gastric dysrhythmias, as unmasked by caloric meal satiety tests (CMST) or water load satiety tests (WLST), represent potential pathophysiological mechanisms related to postprandial symptoms in patients with gastroparesis.

\section{Key Points}

- Satiety testing with caloric or water load acutely evoked symptoms and gastric myoelectrical abnormalities, but these measures did not improve after 24 weeks of insulin therapy.

- To study the effects of continuous subcutaneous insulin infusion with continuous glucose monitoring on GI symptoms, volume consumed, and gastric myoelectrical activity induced by the caloric meal satietytest and water load satiety test.

- Patients with diabetic gastroparesis underwent satiety testing with measures of gastric myoelectrical activity before and after intensive insulin therapy for 24 weeks.

- Volumes of caloric meal and water load, symptoms induced by these two satiety meals, and gastric myoelectrical activity were not improved by intensive insulin therapy.

The effects of intensive insulin treatment on meal-related symptoms and GMA have not been studied in patients with diabetic gastroparesis. Thus, the aims of the current study were to determine the effect of the CMST and WLST on upper GI symptoms and GMA in well-characterized patients with diabetic gastroparesis before and after intensive, open-label, continuous subcutaneous insulin 
infusion (CSII) therapy and continuous glucose monitoring (CGM) for 24 weeks. The patients were enrolled in the Gastroparesis Clinical Research Consortium study protocol GLUMIT-DG and had monthly clinic visits to assess glucose levels, adjust insulin doses, and review diets in order to assess safety of the intensive insulin treatment approach. ${ }^{9}$ We hypothesized that the CMST and WLST would evoke upper gastrointestinal symptoms and gastric dysrhythmias in patients with diabetic gastroparesis and that the ingested volumes, the meal-induced symptoms, and GMA would improve if better glucose control was achieved during the 24 weeks of intensive CSII with CGM.

\section{2 | METHODS}

\section{1 | Patients}

Forty-five patients with diabetic gastroparesis who participated in the GLUMIT-DG study were studied. The patients were recruited from seven centers of the GpCRC. Patients had symptoms for $\geq 1$ year with GCSI scores $>18$. Subjects had upper endoscopy within 1 year to exclude other reasons for symptoms. Gastroparesis was confirmed with gastric scintigraphy before registration with $>60 \%$ meal retention at 2 hours and/or $>10 \%$ at 4 hours. ${ }^{10}$ Type 1 diabetes versus type 2 diabetes was determined by the site investigator based on patient history and review of records.

\subsection{Continuous subcutaneous insulin infusion and continuous glucose monitoring protocol}

Patients were instructed in operating the CSII device (MiniMed Paradigm ${ }^{\circledR}$ Model 722 or Model 723 , Medtronic) coupled with the MiniLink ${ }^{\mathrm{TM}}$ REAL-Time Transmitter CGM system (Medtronic). Subjects needed to show competency in (a) checking glucose levels with CGM plus finger stick methods $\geq 4 \times$ daily, (b) managing CSII including adjusting insulin dosing as described below, and (c) electronically transferring CGM data from their home computer to the GLUMIT-DG study staff every 2 weeks (CareLink, Medtronic). After successful completion of the Run-In, patients were formally enrolled in GLUMIT-DG and permitted to progress to the 24-week treatment and follow-up phase. ${ }^{9}$

\section{3 | Electrogastrography}

After an overnight fast, the subjects had a finger stick glucose level to confirm glucose was $<270 \mathrm{gm} / \mathrm{dL}$. If glucose was over $270 \mathrm{mg} / \mathrm{dL}$, then the glucose level was treated or the test was rescheduled. Subjects were seated in a comfortable chair in a quiet area. EKG-type electrodes were placed in standard position on the upper abdominal surface after the skin was cleaned with alcohol wipes. Electrodes were connected to the electrogastrogram (EGG) recording device (3CPM Company). The EGG signal was digitized for computer analysis. ${ }^{7,11}$ Patients had a 15-minute baseline EGG recording followed by one of the two satiety test protocols listed below. Patients returned on another day for the other of the two EGG with satiety test protocols.

The percentage distribution of GMA power in the four frequency ranges listed below was averaged for Time 0 (before meal ingestion) and for the 10-minute periods after ingestion of the WLST or CMST for group analyses at baseline and 24 weeks. An EGG clinical diagnosis was also determined for the WL test for each subject by comparing values from historical subjects. ${ }^{7}$ The individual EGG diagnoses were as follows: normal (2.5-3.5 cpm), tachygastria (3.5-10 cpm), bradygastria (1-2.5 cpm), mixed gastric dysrhythmia (a combination of tachygastria and bradygastria), and duodenal-respiration (10-15 cpm). These diagnoses were based on the GMA response to the $\mathrm{WL}$ as determined by the percentage distribution of GMA power in the four frequency ranges in response to WLST. ${ }^{7}$ EGG recordings from the clinical centers were reviewed and edited at one site (Wake Forest) by one of the authors (KK) who was blinded to the study site and to baseline or Week 24 visit.

\section{4 | Provocative liquid test meals protocols}

\subsection{1 $\quad$ Water load satiety test}

Subjects ingested water until they achieved the sensation of "completely full" during a five-minute time period. ${ }^{7}$ The volume of water ingested was recorded. The subjects indicated the intensity of fullness, hunger, abdominal discomfort, bloating, and nausea on a $100 \mathrm{~mL}$ visual analog scale (VAS) before and 10,20 , and 30 minutes after the water was ingested. GMA was recorded for 15 minutes before the WL was ingested and for 30 minutes afterward using electrogastrography methods.

\subsection{2 | Caloric meal satiety test}

Subjects ingested $150 \mathrm{~mL}$ of Ensure ${ }^{\circledR}$ every four minutes with no time limit until they were "completely full". ${ }^{11}$ The volume of Ensure ${ }^{\circledR}$ ingested was recorded. The subjects used a $100 \mathrm{~mL}$ VAS to rate the symptoms noted above before and $10,20,30$, and 60 minutes after the CMST. GMA was recorded for 15 minutes for the $\mathrm{CM}$ and for 60 minutes afterward using electrogastrography methods.

\section{5 | Statistics}

Analyses of gastrointestinal symptoms (fullness, hunger, bloating, abdominal discomfort, and nausea) and percentage GMA in the four frequency ranges were stratified by CMST and WLST satiety test results. Changes in meal volume between baseline and 24 weeks were assessed using the paired $t$ test. For comparisons within the WLST, symptoms and GMAs used one presatiety test time and three postsatiety test times at 10,20 , and 30 minutes. For comparisons within the CMST, symptoms used one presatiety test time and four postsatiety test times at 10, 20, 30, and 60 minutes, whereas GMAs used one presatiety test time and six postsatiety 
TAB LE 1 Demographic and clinical characteristics of patients with diabetic gastroparesis

\begin{tabular}{|c|c|}
\hline & Total $n=45$ \\
\hline \multicolumn{2}{|l|}{ Demographics } \\
\hline Female & $31(69 \%)$ \\
\hline White & 37 (82\%) \\
\hline Hispanic & $8(18 \%)$ \\
\hline \multicolumn{2}{|l|}{ Other } \\
\hline Age (y) & 45 (12\%) \\
\hline College graduate & $14(31 \%)$ \\
\hline \multicolumn{2}{|l|}{ Metabolic } \\
\hline$\%$ Type $1 /$ Type 2 Diabetes & $69 \%$ \\
\hline Time since diabetes diagnosis $(y)$ & $21(11)$ \\
\hline Hemoglobin A1c (\%) & $9.4(1.4)$ \\
\hline Weight (kg) & $78(21)$ \\
\hline Body mass index $\left(\mathrm{kg} / \mathrm{m}^{2}\right)$ & $29(8)$ \\
\hline \multicolumn{2}{|l|}{ Gastric emptying scintigraphy } \\
\hline 2-hour gastric retention (\%) & $60(20)$ \\
\hline 4-hour gastric retention (\%) & $30(20)$ \\
\hline \multicolumn{2}{|l|}{ GCSI (Mean \pm SD) } \\
\hline \multicolumn{2}{|l|}{ Nausea } \\
\hline Nausea component & $3.5(1.2)$ \\
\hline Retching component & $2.4(1.6)$ \\
\hline Vomiting component & $2.3(1.9)$ \\
\hline Sub score & $8.1(4.2)$ \\
\hline \multicolumn{2}{|l|}{ Fullness or early satiety } \\
\hline Stomach fullness component & $3.9(0.9)$ \\
\hline Not able to finish component & $3.3(1.3)$ \\
\hline Feeling excessively full component & $3.9(1.0)$ \\
\hline Loss of appetite component & $3.0(1.6)$ \\
\hline Subscore & $14.1(3.6)$ \\
\hline \multicolumn{2}{|l|}{ Bloating } \\
\hline Bloating component & $3.7(1.2)$ \\
\hline Stomach visibly larger component & $3.4(1.4)$ \\
\hline Subscore & $7.1(2.3)$ \\
\hline Total GCSI & $29.3(7.1)$ \\
\hline
\end{tabular}

test times at 10, 20, 30, 40, 50, and 60 minutes. Comparisons between the WSTL and CMST used time tests that were common to both, namely the one presatiety test time and the three postsatiety test times at 10, 20, and 30 minutes. Mean scores for symptoms and GMAs compared the baseline vs 24 -week visit assessed at the presatiety and postsatiety test times. Changes in scores for symptoms and GMAs compared presatiety vs postsatiety test times separately at the baseline and 24-week visits. Repeated measures were analyzed using linear regression with mixed effects. $P$ values $\leq .05$ were considered statistically significant. The data analysis for this paper was generated using both SAS ${ }^{12}$ and Stata software. ${ }^{13}$

\section{3 | RESULTS}

\section{1 | Patients}

The patients were 31 women and 14 men ages 18 to 70 years. Thirty-one patients had type 1 diabetes and 14 had type 2 diabetes. Characteristics of the patients are shown in Table 1. Duration of diabetes averaged $21 \pm 11$ years. Body mass index averaged 29. At baseline $\mathrm{HbA} 1 \mathrm{c}$ averaged $9.4 \% \pm 1.4 \%$. The average percent meal retained at two hours was $63 \% \pm 20 \%$ and four hours was $32 \% \pm 20 \%$. At four hours 16 patients had severe retention (>30\% retained), five had moderate retention (20\%-30\% retained), and 15 had mild retention (10\%-20\% retained). GCSI averaged $29.3 \pm 7.1$ (maximum score of 45 ). Almost half of the patients were taking prokinetic agents, and $70 \%$ were taking a proton pump inhibitor. The T2DM patients were significantly older, had higher BMI, used less continuous insulin pump therapy, used more antidiabetic medications other than insulin, and had ingested lower mean volumes during the satiety water load and liquid nutrient tests compared to T1DM patients. Other demographic and standard laboratory results were similar in the two groups as previously published. ${ }^{9}$

Results of treatment with CSII with CGM for 6 months increased time in euglycaemia $(70-180 \mathrm{mg} / \mathrm{dL})$ from $44 \%$ to $52 \%(P<.01)$, decreased time in hypoglycemia ( $<70 \mathrm{mg} / \mathrm{dL}$ ) (from $3.9 \%$ to $1.8 \%$ ) and hyperglycemia (>300 mg/dL; from $14.2 \%$ to $6.9 \%, P<.0001$ ). HbA1c was reduced from $9.4 \%$ to $8.3 \%$ or $1.1 \% \pm 1.2 \%(P<.01)$ were previously reported. ${ }^{9}$ Gastric symptom scores decreased $23 \%(P<.0001)$ with lower nausea/vomiting (35\%), fullness/early satiety (17\%), and bloating/distention (21\%) sub-scores ( $P \leq .002)$. Quality-of-life scores improved $29 \%(P<.0001)$. Satiety test tolerance increased $14 \%(P=.05)$. HbA1c decreased more in patients with type 2 diabetes $(\mathrm{T} 2 \mathrm{DM})(2.0 \% \pm 2.1 \%$ vs $0.7 \% \pm 1.1 \%, P=.002)$ but symptoms improved more in patients with type 1 diabetes (T1DM) (32\% vs $1 \%$, $P=.01)$. Six severe hypoglycemic events occurred on treatment versus one during screening (0.3 vs 0.1 /person-year, $P=.23$ ). ${ }^{9}$

Of the 45 subjects in the current study, 43 and 44 subjects had EGGs with WLSTs and CMSTs, respectively, at baseline. At 24 weeks, 37 and 39 subjects had EGGs with WLSTs and CMSTs, respectively. EGGs were not obtained or analyzed at 24 weeks in 11 patients because (a) patients were not able to return for either the water load test or the satiety test, which were performed on separate days, or (b) movements of the arms, legs, or torso resulted in off scale deflections of the EGG signal. EGG recordings with excessive movement artifact were not analyzed.

\subsection{Volumes ingested during the water load satiety test and caloric meal satiety test at baseline and 24 weeks}

The average volume of water ingested was $437 \pm 216 \mathrm{~mL}$ at baseline and was $413 \pm 238$ at 24 weeks $(P=.56$; Figure 1$)$. Volumes of water ingested in the five-minute time limit ranged from 150 to 
$1150 \mathrm{~mL}$. Historical control subjects ingested water until completely full using the same protocol, and the average volume ingested was $648 \pm 205 \mathrm{~mL} .{ }^{14}$ Thus, the lower limit of normal was set at $>238 \mathrm{~mL}$ water ingested in five minutes. Using this cutoff, $24 \%$ of patients had abnormal WLSTs at baseline and $27 \%$ at 24 weeks.

The average volume ingested during the CMST was $427 \pm 287 \mathrm{~mL}$ at baseline and was $480 \pm 217 \mathrm{~mL}$ at 24 weeks $(P=.44$; Figure 1$)$. Volumes ingested by our subjects ranged from 125 to $1422 \mathrm{~mL}$ to achieve the sense of "completely full." The duration of ingestion ranged from 4 to 10 minutes. Historical control subjects ingested 800 to $1500 \mathrm{~mL}$ of a caloric load over unlimited time to achieve maximum satiety using similar CMST protocols. ${ }^{4-6}$ The CMST with a protocol similar to the current study reported healthy subjects ingest $1048 \pm 421 .{ }^{5}$ Thus, the lower range of normal was set at $\geq 206 \mathrm{~mL}$ ingested. Using this cutoff, $15 \%$ and $19 \%$ of the diabetic subjects ingested $<206 \mathrm{~mL}$ at baseline and at 24 weeks, respectively.

\subsection{Symptoms and GMA in response to the water load and caloric meal satiety tests at baseline and 24- week visits}

At baseline and 24 weeks fullness increased and hunger decreased significantly and bloating and abdominal discomfort increased significantly after the WLST (Ps < 0.02; Figure 2). Nausea increased significantly after WLST at Week $24(P<.01)$ but not at baseline $(P=.08)$. There were no differences in the intensity of these symptoms at 24 weeks compared with baseline.

Figure 3 shows GMA results in the bradygastria, normogastria, tachygastria, and duodenal frequencies before (Time 0) and 10, 20, and 30 minutes after the subjects ingested water until they were completely full at baseline and 24-week visits. The percentage of normal $3 \mathrm{cpm}$ GMA and tachygastria increased after the WLST at each visit but changes were not statistically significant. There were no changes in the average percentage distribution of GMA in these four frequency ranges at Week 24 compared with baseline values.

Figure 4 shows fullness, hunger, bloating, abdominal discomfort, and nausea before and after the CMST test. Compared with premeal, fullness increased and hunger decreased significantly and bloating, abdominal discomfort, and nausea increased significantly after the CMST (Ps < 0.01). These latter three symptoms remained significantly elevated for 60 minutes after the meal. There were no significant differences in the intensity of symptoms elicited by the CMST after 24 weeks of CSII therapy compared with the baseline visit.

Figure 5 shows GMA in the bradygastria, normogastria, tachygastria, and duodenal frequencies before and after the CMST. The percentage distribution of GMA decreased significantly in the bradygastria range and increased significantly in the normal $3 \mathrm{cpm}$ range at both visits. The percentage distribution of GMA in the tachygastria range increased significantly $(P=.002)$ at 24 weeks only. There were no significant differences in the GMA response to the CMST when the Week 24 visit was compared with baseline.

\subsection{Comparison of symptoms and GMA after water load and caloric meal satiety tests}

At baseline, nausea intensity was increased similarly after ingestion of the CMST and WLST $(P=.75)$. CMST evoked more fullness, less hunger, and more bloating and abdominal discomfort compared with the WL test (Ps < 0.01). After the CMST, bradygastria decreased and tachygastria increased significantly more compared with WLST (Ps < 0.03). The normal $3 \mathrm{cpm}$ GMA responses to WLST and CMST were similar $(P=.41)$.

At Week 24, fullness, bloating, abdominal discomfort, and nausea were significantly greater after CMST and hunger significantly less compared with WLST (Ps < 0.01). Bradygastria was decreased and tachygastria increased after CMST compared with WLST at
FIGURE 1 Volumes ingested by subjects during the $(A)$ water load satiety test (WLST) and (B) caloric meal satiety test (CMST) test meals until they were completely full. The $X$ axis shows baseline and Week 24 visits and the $Y$ axis shows the volume in milliliters $(\mathrm{mL})$ ingested at baseline and $24 \mathrm{wk}$ after insulin pump therapy and continuous glucose monitoring. The volume of water ingested at baseline was $437 \mathrm{~mL}$ and $413 \mathrm{~mL}$ at 24 wk $(P=.86)$. The average volume of caloric test meal ingested at baseline was $427 \mathrm{~mL}$ and $480 \mathrm{~mL}$ at Week $24(P=.44)$
(A)

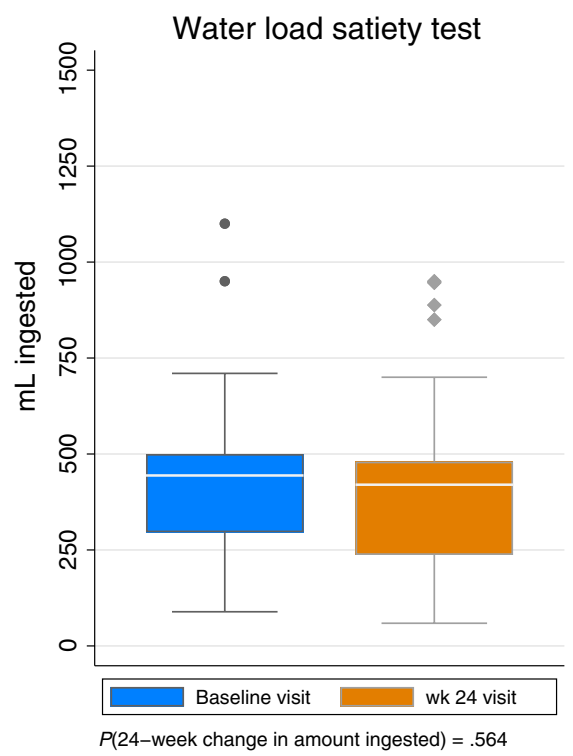

(B)

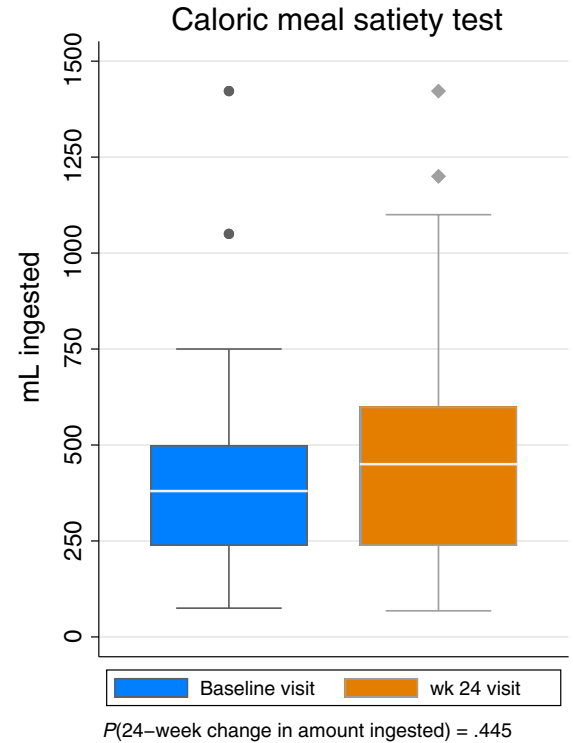



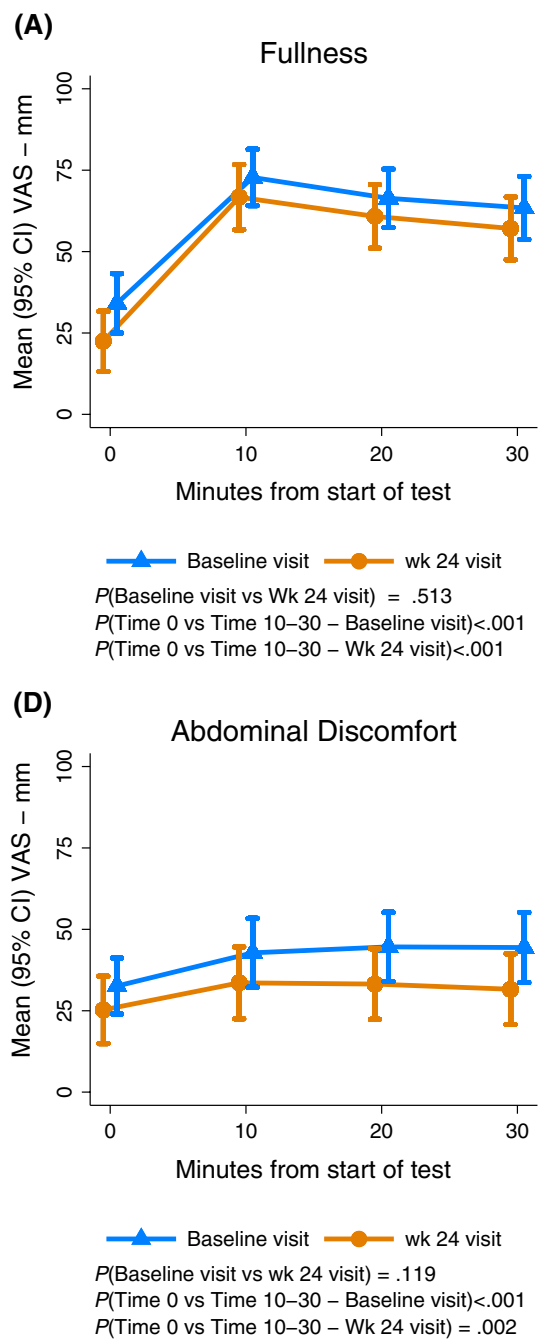

(B)

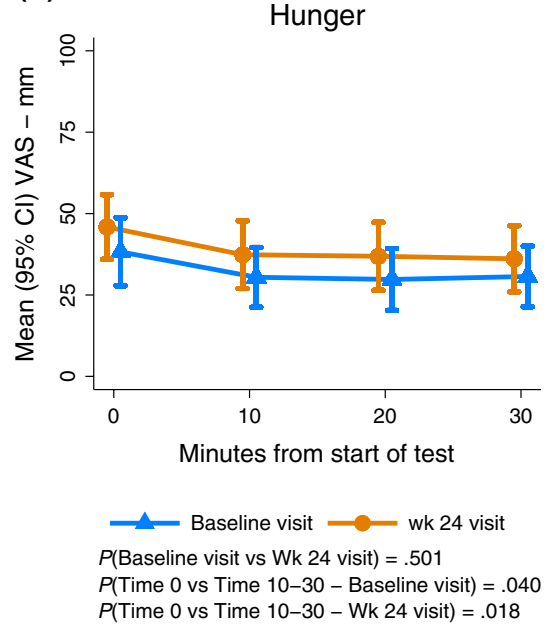

(E)

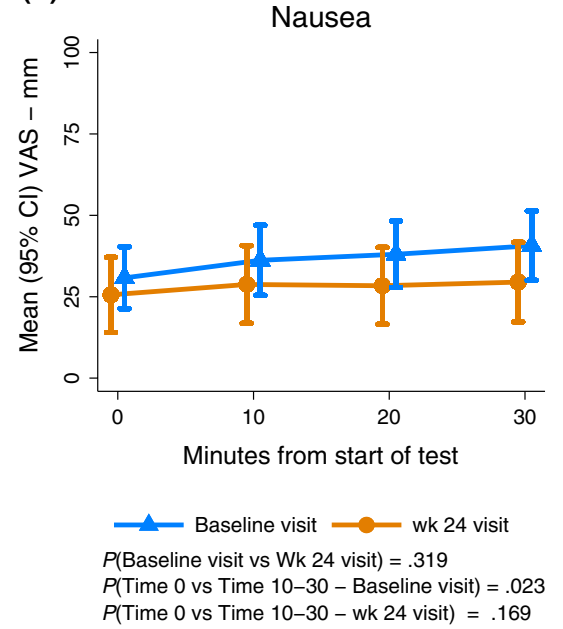

(C)

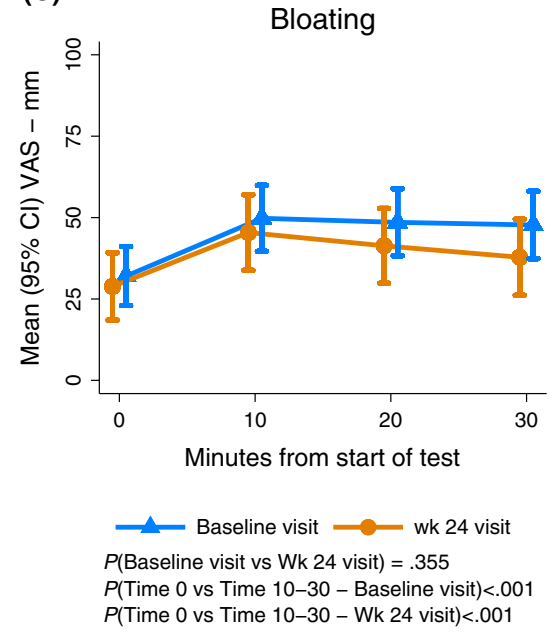

FIG URE 2 Fullness (A), hunger (B), bloating (C), abdominal discomfort (D), and nausea (E) scores are shown before and after the water load satiety test (WLST) test at baseline and at $24 \mathrm{wk}$. The $X$ axis shows time in minutes. Time 0 indicates the 10 min before the test meal and 10,20, and 30 min indicate time after ingestion. The $Y$ axis shows the symptom intensity on the visual analog scale (VAS) in millimeters $(\mathrm{mm})$. Fullness increased significantly after the WLST and hunger decreased significantly at baseline and Week 24 visits. Symptoms of bloating and abdominal discomfort and nausea all increased significantly at the baseline visit, and bloating and abdominal discomfort increased significantly at 24 wk. Compared with the baseline visit, there were no significant changes in symptoms at Week 24

Week 24 (Ps < 0.001). Normal 3 cpm GMA responses were similar after WLST and CMST $(P=.29)$.

\subsection{Clinical EGG diagnoses after water load satiety test at baseline and 24 weeks}

Thirty-one of the 45 patients had the EGG and WLST at baseline and Week 24 (Tables 2 and 3). The overall clinical EGG diagnoses were as follows: At baseline the EGG was normal in 14 subjects (45\%) and abnormal in 17 subjects (six had tachygastria, four had bradygastria, three had mixed dysrhythmias, four had duodenalrespiration pattern). At 24 weeks, 10 subjects (32\%) had normal $3 \mathrm{cpm}$ GMA and 20 (68\%) had dysrhythmias (10 had tachygastria, three had bradygastria, six had mixed dysrhythmias, one had the duodenal-respiration pattern). The agreement in EGG diagnoses between baseline and 24-week visits was 55\%. Five of these 31 subjects (16\%) had normal $3 \mathrm{cpm}$ GMA at both baseline and at 24 weeks. Figure 6 shows an example of normal 3 cpm GMA before and after the WLST and consistent $3 \mathrm{cpm}$ peaks in the running spectral analysis of the GMA in a patient with diabetic gastroparesis. Figure 7 shows gastric dysrhythmias before and after the WLST and peaks in the tachygastria range in the running spectral analysis in another patient.

\section{4 | DISCUSSION}

Symptoms and physiological measures immediately after meals have been infrequently studied in patient with diabetic gastroparesis. On the other hand, satiety tests with barostat recordings have been studied frequently in patients with functional dyspepsia 
(A)

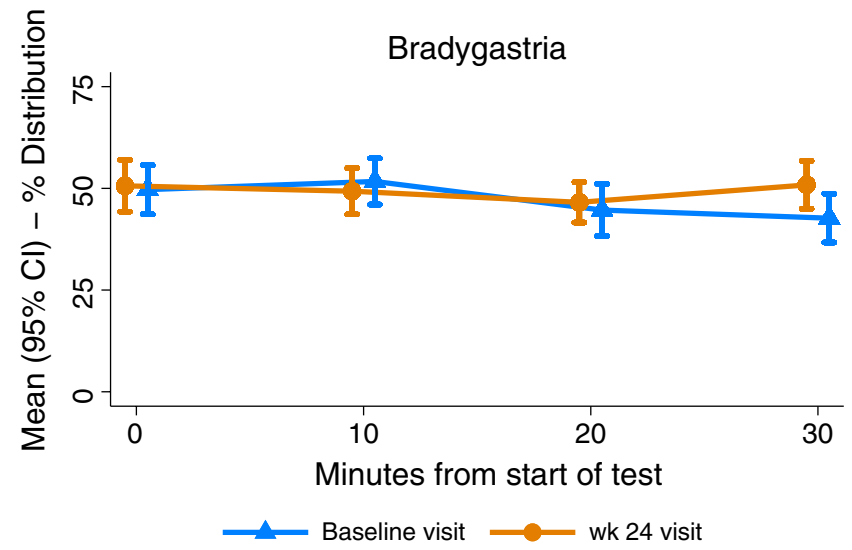

$P($ Baseline visit vs Wk 24 visit $)=.104$

$P($ Time 0 vs Time $10-30-$ Baseline visit $)=.287$

$P($ Time 0 vs Time $10-30-$ Wk 24 visit $)=.644$

(C)

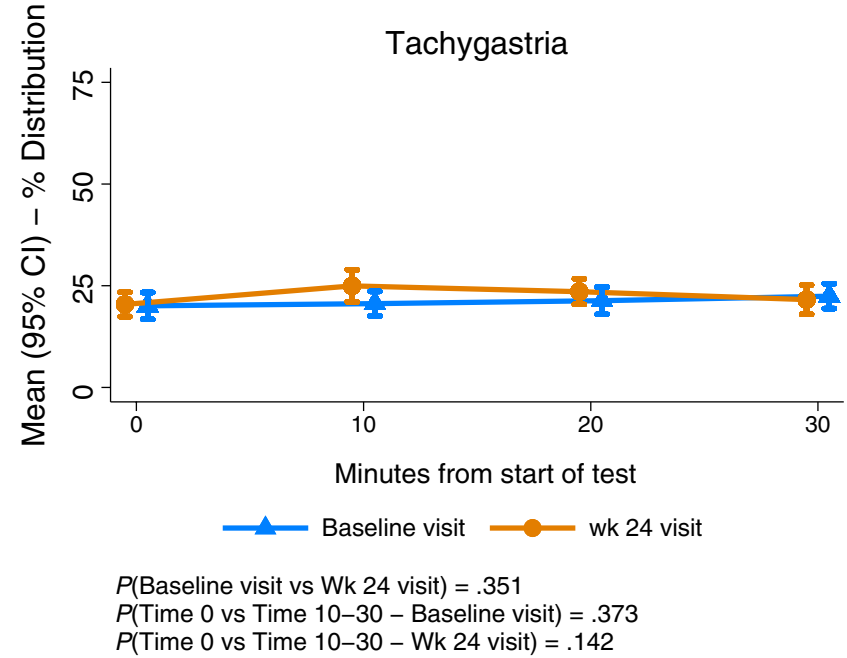

(B)

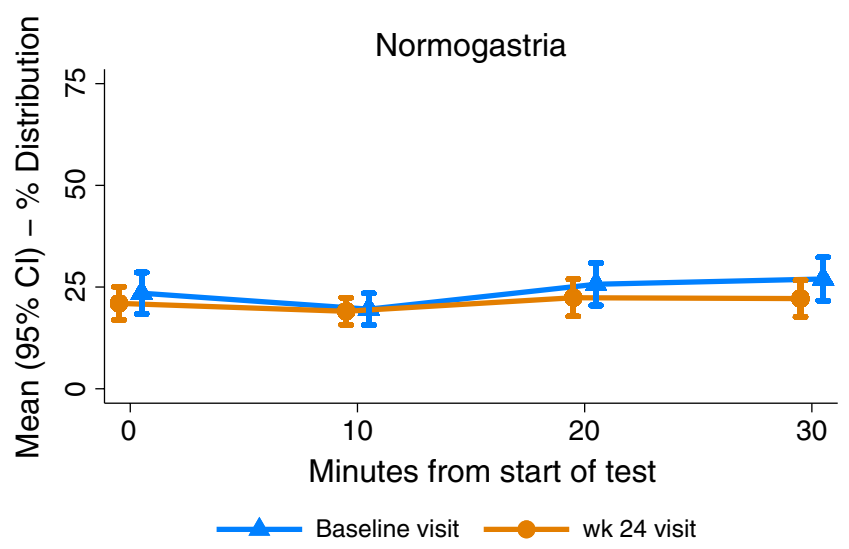

$P($ Baseline visit vs Wk 24 visit $)=.462$

$P($ Time 0 vs Time $10-30-$ Baseline visit $)=.816$

$P($ Time 0 vs Time $10-30-$ Wk 24 visit $)=.927$

(D)

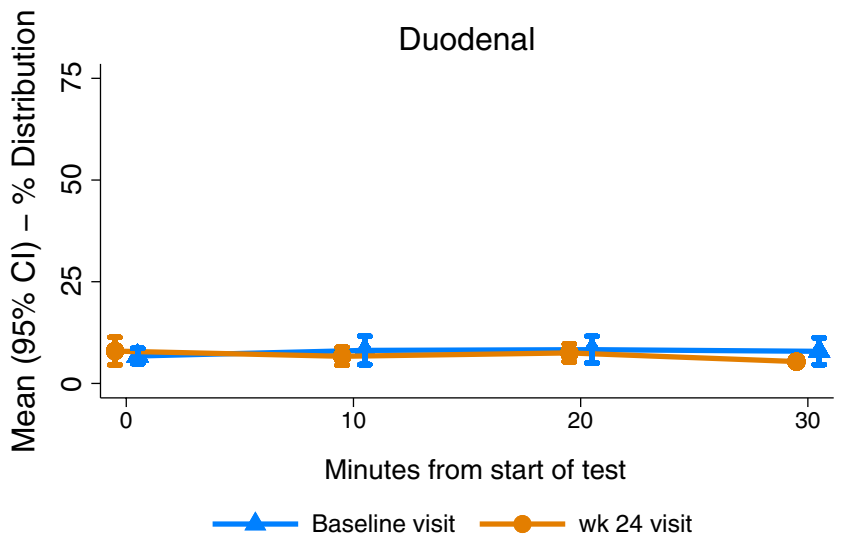

$P($ Baseline visit vs Wk 24 visit $)=.539$

$P($ Time 0 vs Time $10-30-$ Baseline visit $)=.256$

$P($ Time 0 vs Time $10-30-$ Wk 24 visit $)=.310$

FIGURE 3 Gastric myoelectrical activity (GMA) before and after the water load satiety test at baseline and at 24 wk. Bradygastria (1.0-2.5 cpm) (A), normogastria (2.5-3.5 cpm) (B), tachygastria (3.5-10 cpm) (C), and duodenal-respiration (10-15 cpm) (D) frequency ranges are shown. The $X$ axis indicates time in minutes with Time 0 indicating the 10 min before the test meal and the 10, 20, and 30-minute periods after ingestion. The $Y$ axis indicates the percent distribution of GMA power in the four frequency ranges. Normogastria and tachygastria increased 20 and 30 min after ingestion at baseline and Week 24 visits, but changes were not statistically significant. Compared with baseline there were no significant changes in GMA at Week 24

who ingest low volumes of the meals in association with decreased accommodation and/or gastric hypersensitivity. ${ }^{4-6}$ In the current study, almost $30 \%$ of our diabetic subjects ingested $<236 \mathrm{~mL}$, which is $<2$ SD below the mean in healthy controls. ${ }^{5,14}$ Karamanolis et al found symptoms evoked with a $200 \mathrm{~mL}$ test meal correlated better with gastroparesis symptoms than the rate of gastric emptying or gastric accommodation in patients with idiopathic gastroparesis. ${ }^{2}$ Gastroparesis patients who consumed small volumes ( $<250 \mathrm{~mL}$ ) during the WLST had increased GCSI scores, especially nausea, and more severe delays in gastric emptying compared with patients who consumed normal volumes $(>557 \mathrm{~mL}){ }^{8}$ Nausea, bloating, and abdominal discomfort were reported within minutes after the caloric meal or water load were ingested. Possible mechanisms include sensitivity to gastric distention, abnormalities in gastric accommodation, or both. However, these possible abnormalities were not investigated in this study.

The normal $3 \mathrm{cpm}$ GMA is associated with normal numbers of gastric ICCs, the pacemaker cells that generate $3 \mathrm{cpm}$ slow waves and coordinate gastric peristalsis during normal gastric emptying. ${ }^{15,16}$ Studies have suggested that the majority of diabetic patients with gastroparesis have severe depletion of ICCs in the corpus and antrum (0-3 ICCs/hpf) and gastric dysrhythmias ranging from tachygastria to bradygastria. ${ }^{15,16}$ Distention of the antrum with a balloon was associated with more upper GI symptoms and more gastric dysrhythmias compared with distention of the fundus in healthy subjects. ${ }^{17}$ In our study, gastric dysrhythmias were evoked after ingestion of volumes of water in most patients and included tachygastria, bradygastria, and mixed gastric 
(A)

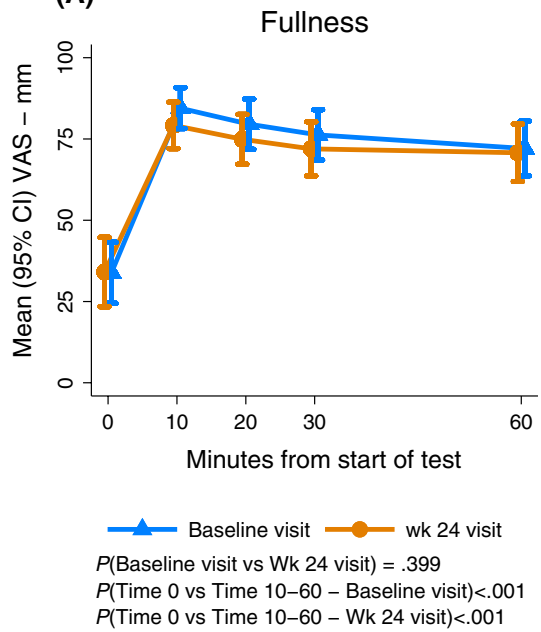

(D)

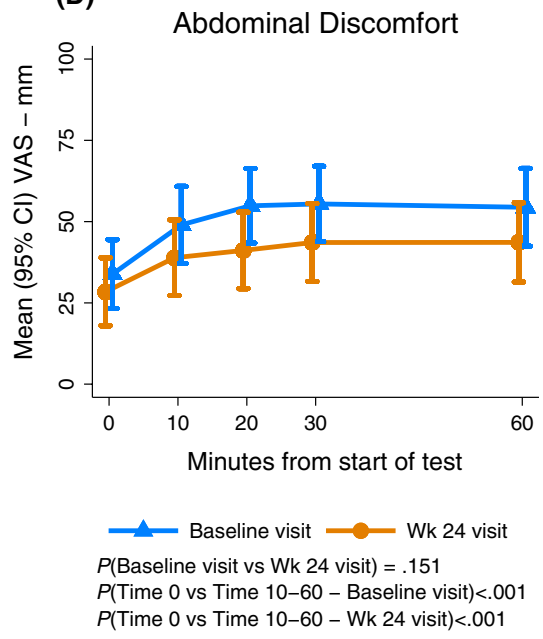

(B)

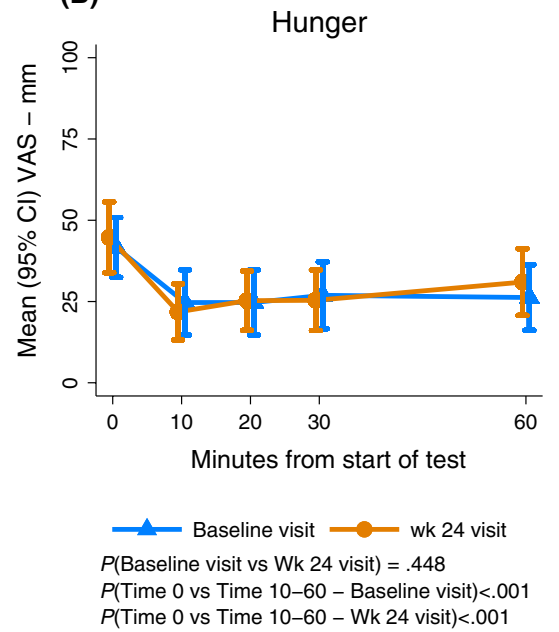

(E)

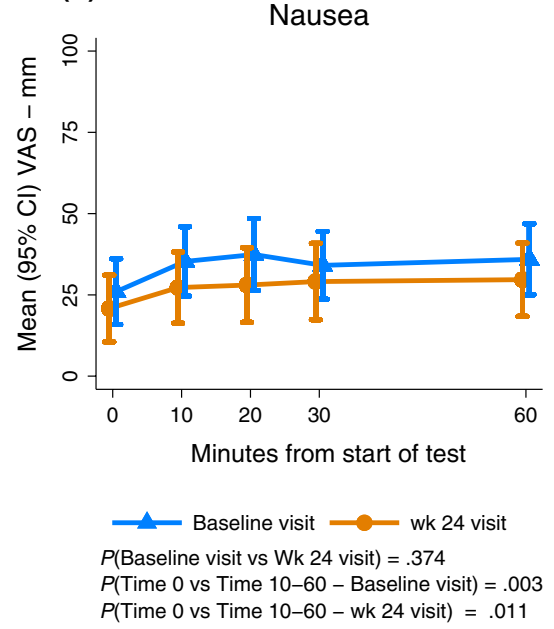

(C)

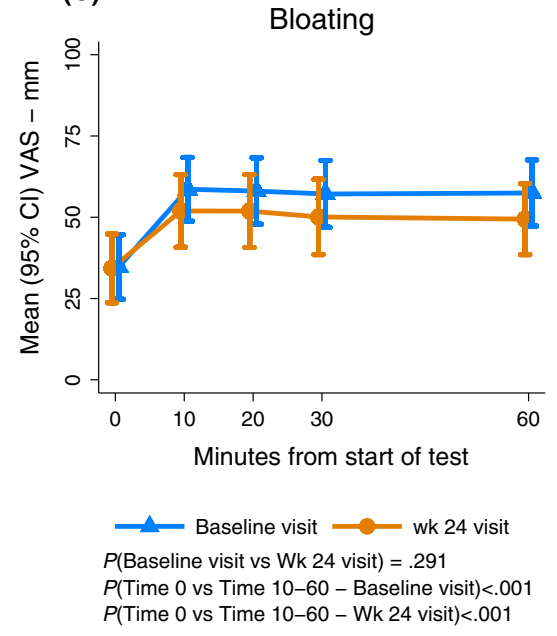

FIGURE 4 Fullness (A), hunger (B), bloating (C), abdominal discomfort (D), and nausea (E) scores are shown before and after the caloric meal satiety test (CMST) at baseline and $24 \mathrm{wk}$. The $X$ axis shows time in minutes. Time 0 indicates the 10 min before the test meal and 10 , 20,30, and 60 min indicates time after ingestion. The $Y$ axis shows the symptom intensity on the visual analog scale (VAS) in millimeters (mm). Fullness increased significantly after the CMST and hunger decreased significantly, and symptoms of bloating, abdominal discomfort, and nausea all increased significantly at baseline and the Week 24 visits. Compared with baseline visits, there were no significant changes in symptoms at Week 24

dysrhythmias. Symptoms and changes in $3 \mathrm{cpm}$ GMA increased after the CMST to a greater extent than with the WLST. Thus, distention of the antrum by liquid test meals in our patients with diabetic gastroparesis was associated with changes in GMA and acute postprandial symptoms. Gastric dysrhythmias are also elicited when glucose levels are over $220 \mathrm{mg} / \mathrm{dL}$ in normal subjects ${ }^{18}$ and are more prevalent in diabetic patients during hyperglycemia. ${ }^{19}$ Glucose levels $>220 \mathrm{mg} / \mathrm{dL}$ but $<270 \mathrm{mg} / \mathrm{dL}$ on the day of the satiety test meals may have affected the GMA at baseline and follow-up visits.

Sixteen percent of our patients with diabetic gastroparesis had normal $3 \mathrm{cpm}$ GMA in response to WLST at both baseline and at Week 24. In contrast to gastric dysrhythmias, the presence of normal $3 \mathrm{cpm}$ GMA suggests normal function of the gastric ICCs, the pacemaker cells of the stomach. ${ }^{15,16}$ Approximately, $20 \%$ of patients with diabetic gastroparesis had normal numbers of ICCs by immunohistochemical stains from full-thickness biopsies of the stomach corpus, although electron microscopy showed poor ICC-nerve and ICCsmooth muscle contacts in these patients. ${ }^{20}$ Gastroparesis patients with normal $3 \mathrm{cpm}$ GMA may have a fixed or functional obstructive gastroparesis subtype ${ }^{21,22}$ secondary to pyloric dysfunction which may contribute to symptoms and the delay in gastric emptying.

We hypothesized that improvement in $\mathrm{HbA1c}$ after intense insulin therapy would improve symptoms elicited by the satiety tests and GMA. However, after 24 weeks of CSII with CGM, the symptoms evoked by the caloric and non-caloric satiety test meals were similar to baseline. Our previous study showed that intensive treatment with CSII with CGM was associated with a $23 \%$ improvement in GCSI and a 1.1\% decrease in HbA1c (from $9.4 \% \pm 1.4 \%$ to $8.3 \% \pm 1.3 \%$ ), supporting the importance of efforts to improve 

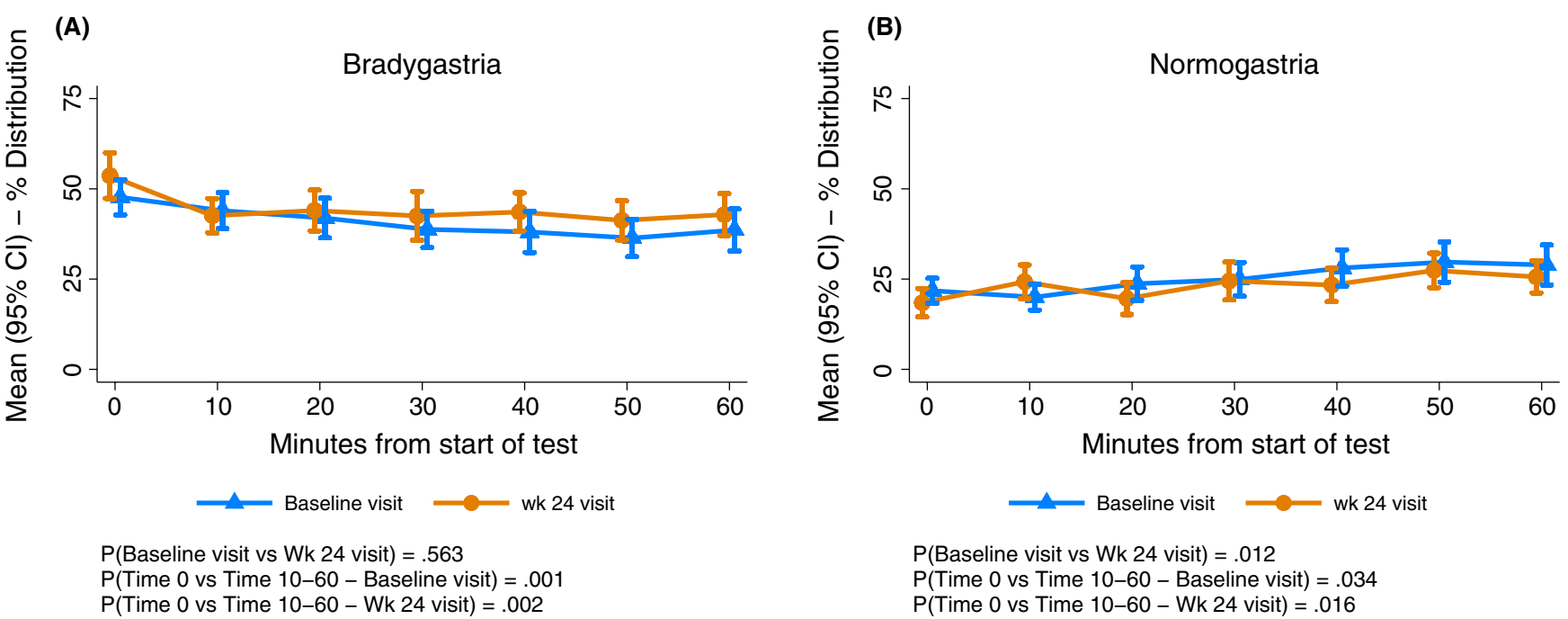

$P($ Time 0 vs Time $10-60-$ Baseline visit $)=001$
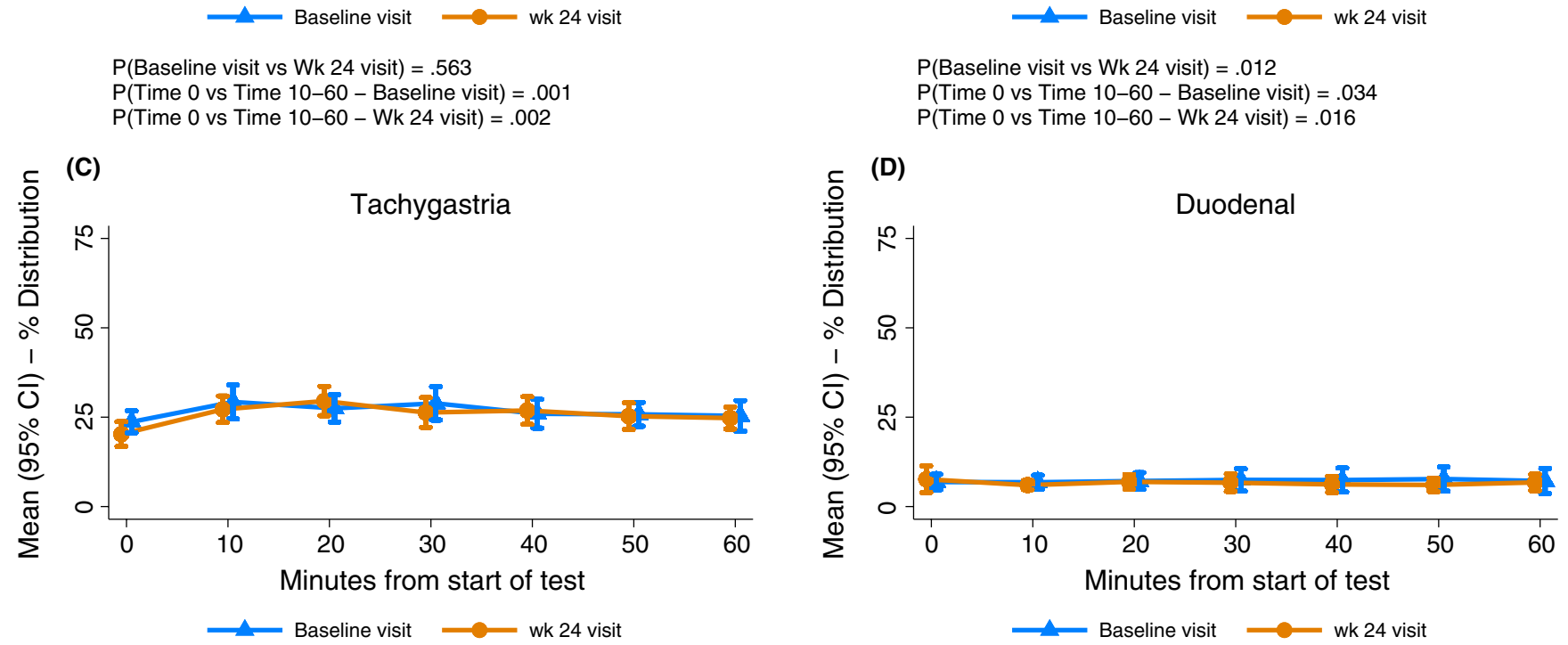

$P($ Baseline visit vs Wk 24 visit $)=.112$

$\mathrm{P}($ Time 0 vs Time $10-60-$ Baseline visit $)=.076$

$\mathrm{P}($ Time 0 vs Time $10-60-$ Wk 24 visit $)=.002$

$\mathrm{P}$ (Baseline visit vs wk 24 visit $)=.738$

$\mathrm{P}($ Time 0 vs Time $10-60-$ Baseline visit $)=.803$

$\mathrm{P}($ Time 0 vs Time $10-60-$ wk 24 visit $)=.461$

FIGURE 5 Gastric myoelectrical activity (GMA) before and after the caloric meal satiety test (CMST) are shown at baseline and at 24 wk. Bradygastria (1.0-2.5 cpm) (A), normogastria (2.5-3.5 cpm) (B), tachygastria (3.5-10 cpm) (C), and duodenal-respiration (10-15 cpm) (D) frequency ranges are shown. The $X$ axis and $Y$ axis are similar to Figure 3 . After ingestion of the caloric meal, normogastria significantly increased and bradygastria significantly decreased at baseline and 24 wk. At 24 wk tachygastria increased significantly after the CMST.

There were no differences in GMA at Week 24 compared with baseline

TAB LE 2 Electrogastrogram (EGG) diagnoses in response to water load satiety test in patients with diabetic gastroparesis at baseline and after 24 weeks of insulin therapy with continuous glucose monitoring

\begin{tabular}{|lcc|}
\hline & Baseline & Week 24 \\
\hline Normal & $14(45)$ & $10(32)$ \\
\hline Tachygastria & $6(19)$ & $11(36)$ \\
\hline Bradygastria & $4(13)$ & $3(10)$ \\
\hline Mixed dysrhythmias & $3(10)$ & $6(19)$ \\
\hline Duodenal-respiration & $4(13)$ & $1(3)$ \\
\hline
\end{tabular}

glycemic control. ${ }^{9}$ However, gastroparesis symptoms reported using GCSI reflect a two-week recall of symptoms and thus reflect an average of symptoms related to daily meals and activities over time. ${ }^{9}$ In the current study, subjects ingested the liquid test meals until they
TABLE 3 Agreement ${ }^{\mathrm{a}}$ in electrogastrogram (EGG) Diagnoses between Baseline and Week 24

\begin{tabular}{llll} 
& \multicolumn{2}{l}{ Week 24} & \\
\cline { 2 - 4 } Baseline & Normal & Abnormal & Total \\
Normal & 5 & 9 & 14 \\
Abnormal & 5 & 12 & 17 \\
Total & 10 & 21 & 31 \\
\hline
\end{tabular}

${ }^{\mathrm{a}} 55 \%$ with Kappa value 0.06 .

were completely full, an acute challenge of gastric neuromuscular function that also allowed collection of meal-induced symptoms in real time with concomitant GMA recordings. Under these test meal conditions, the intense insulin therapy with CGM did not affect symptoms or physiological measures during the WLST or CMST. 
(A)

Baseline water load safety test

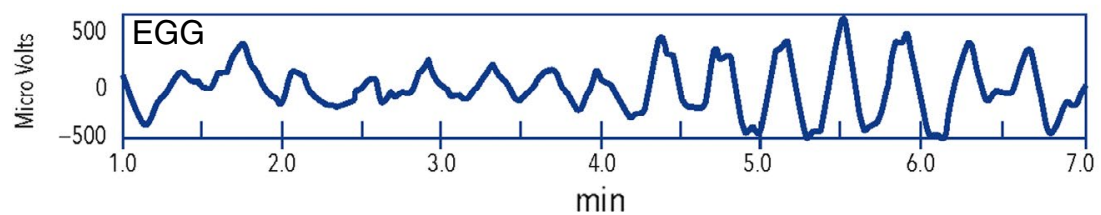

Poststimulation period 1

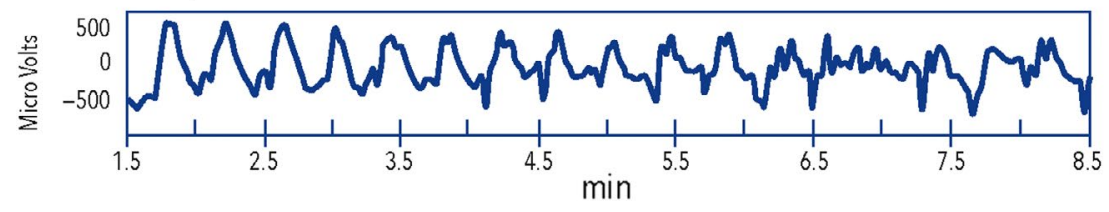

Poststimulation period 2

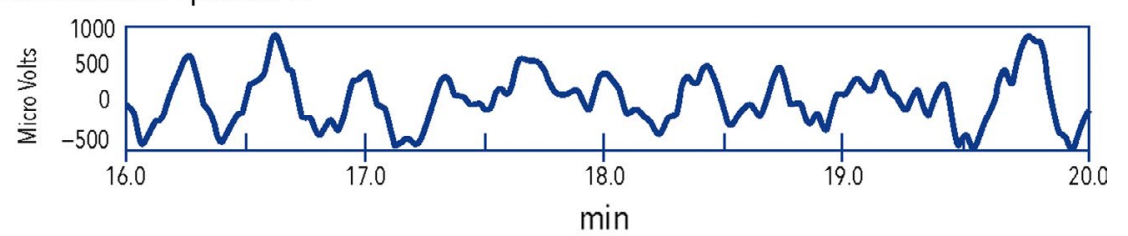

Poststimulation period 3

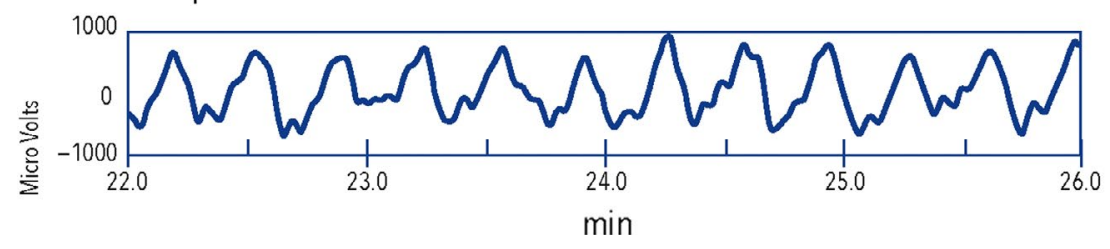

(B)

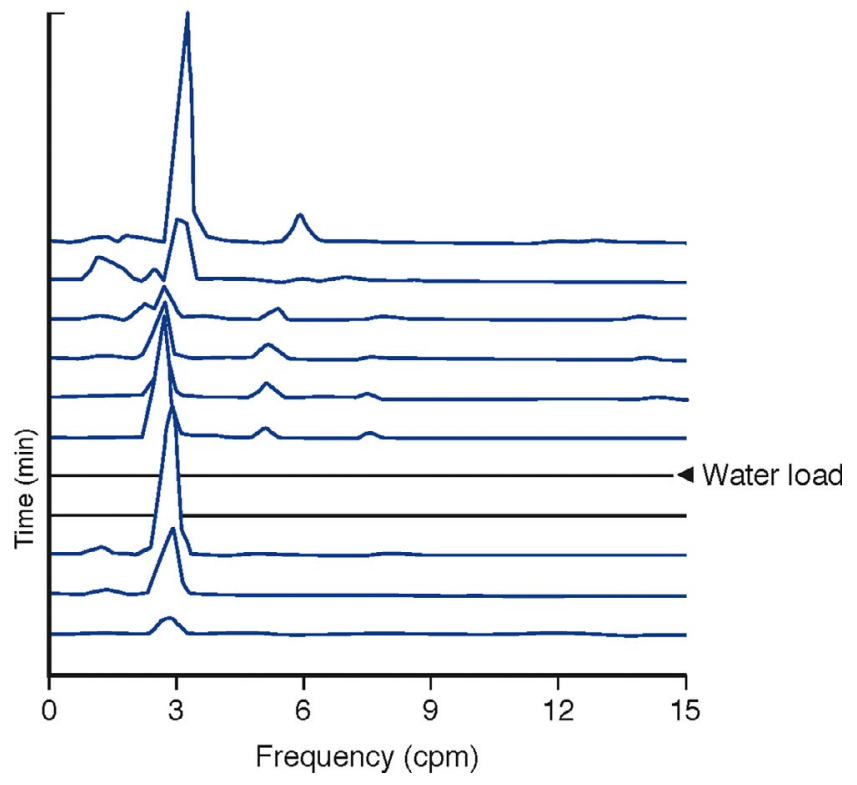

FIGURE 6 GMA rhythm strips from the electrogastrogram recording from a subject with diabetic gastroparesis are shown in A. GMA rhythm strips from baseline (before WLST) and from 10,20 , and 30 min after the WLST (labeled poststimulation period 1, 2, and 3 , respectively). The $X$ axis shows time in minutes and the $Y$ axis shows microvolts. Note the 3 cycle per minute $(\mathrm{cpm})$ waves in GMA at baseline (before water load) and in the rhythm strips from poststimulation period 1,2 , and 3 which are from the 10, 20, and 30-min periods after the WLST. Running spectral analysis presented in $B$ are from the GMA recordings shown in $6 \mathrm{~A}$. The $X$ axis shows frequency from $1-15 \mathrm{cpm}$. The $Y$ axis shows time with each line representing 4 min of GMA with $75 \%$ overlap. The $Z$ axis shows peaks that reflect the frequencies according to amplitude or power of GMA in the electrogastrogram (EGG) signal.
There are several potential reasons for the lack of improvement in symptoms and physiological measures in response to the satiety meal tests: (a) HbA1c decreased $1.1 \%$ from $9.4 \%$ to $8.3 \%$ during the 6-month treatment period, but hypo- and hyperglycemia events still occurred over $40 \%$ of the time as recorded with CGM. ${ }^{9}$ Thus, the overall glycemic control remained abnormal at $8.3 \%$. The $1.1 \%$ improvement in HbA1c may not have been sufficient to positively affect gastric neuromuscular function and symptoms in these patients with diabetic GP; (b) our patients had known diabetes for an average of 21 years. The duration of treatment with CSII with 
FIGURE 7 GMA rhythm strips from an electrogastrogram (EGG) recording from a subject with diabetic gastroparesis are shown in A. GMA rhythm strips from baseline (before WLST) and from 10, 20 , and 30 min after the WLST (labeled poststimulation period 1,2 , and 3 , respectively). The $X$ axis shows time in minutes and the $Y$ axis shows microvolts. Note the lack of 3 cycle per minute (cpm) GMA at baseline and irregular GMA after the WLST in the post stimulation time periods. Running spectral analysis presented in B are from the GMA recordings before and after the WLST shown in 7A. The $X$ axis shows frequency from 1-15 cpm. The $Y$ axis shows time with each line representing 4 min of GMA with $75 \%$ overlap. The $Z$ axis shows peaks that reflect frequencies according to amplitude or power of the GMA in the EGG signal. The two flat lines indicate the time of ingestion of the water load.
(A)

Baseline water load safety test
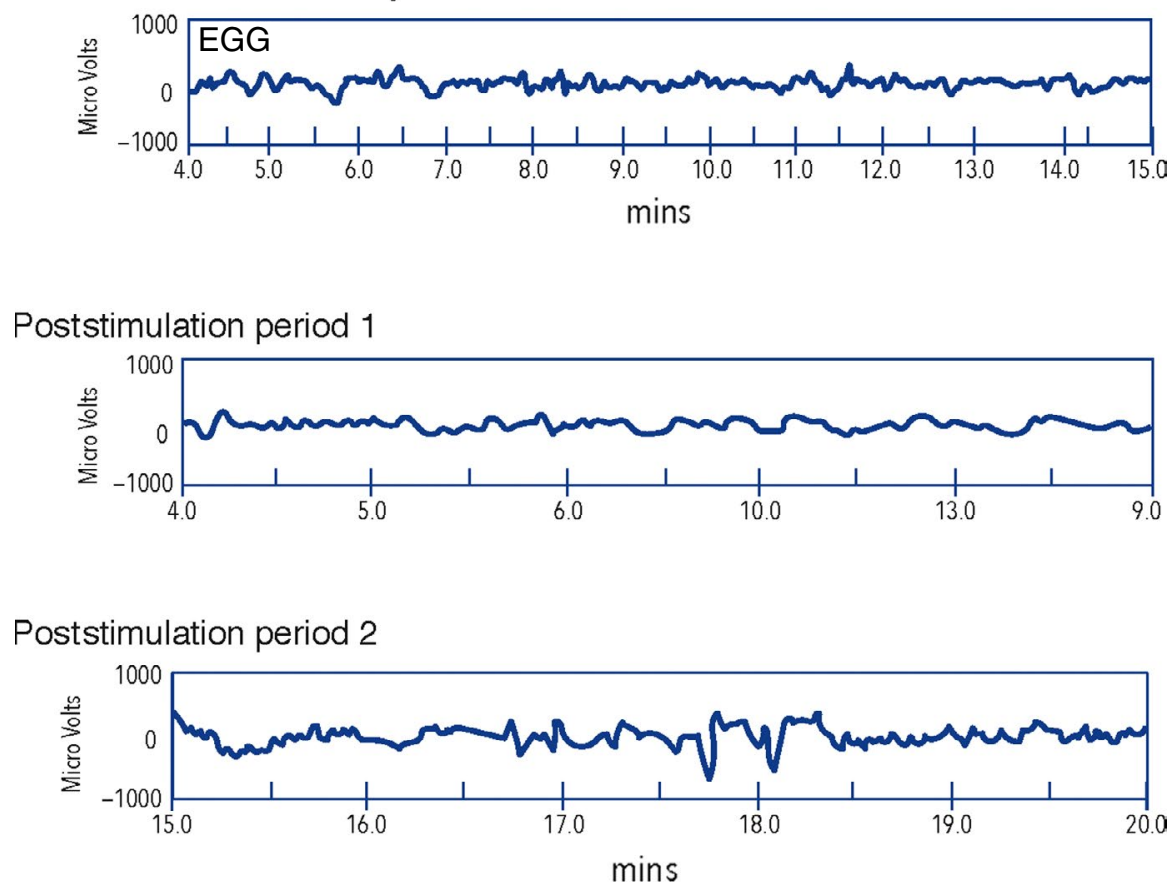

Poststimulation period 3

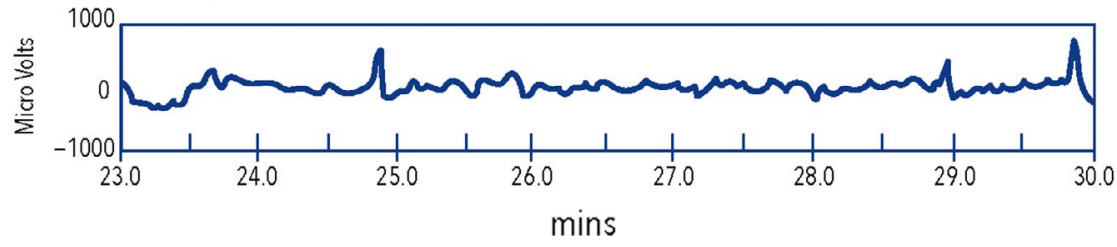

(B)

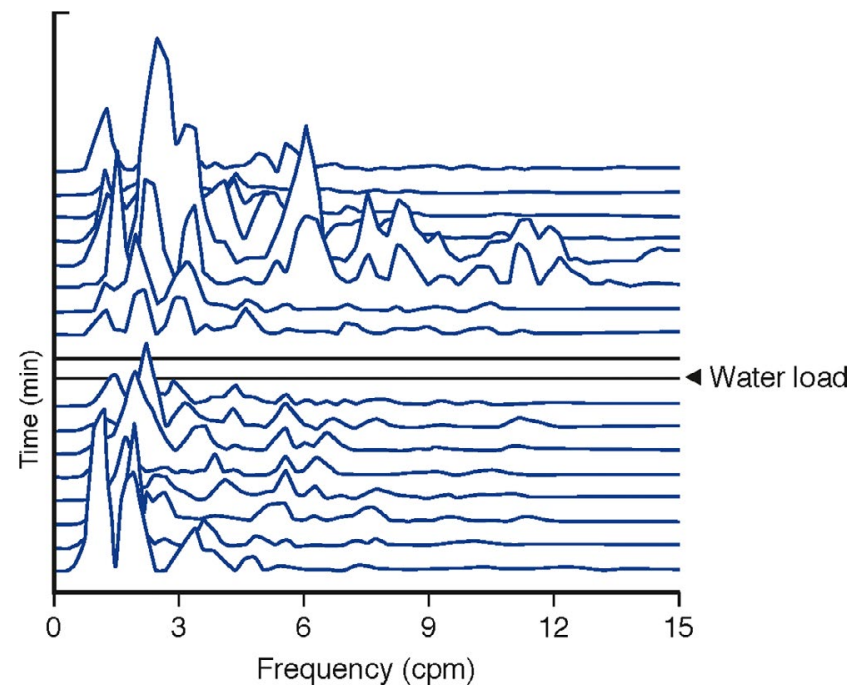

CGM was 6 months, but a longer treatment and better control of glucose may be required to improve poor gastric accommodation, gastric dysrhythmias, and symptoms evoked by the satiety tests in these patients. Improvement in $\mathrm{HbA1c}$ was not associated with improved symptoms or rates of gastric emptying in other studies $^{23,24}$; (c) factors other than glycemic control may be important in meal-induced symptoms and GMA abnormalities in diabetic gastroparesis. Hyperglycemia alone was not enough to cause gastroparesis in diabetic mice. In addition to hyperglycemia, a switch from M2 macrophages to M1 macrophages in the circular muscle layer and in the myenteric plexus of the stomach was required to develop gastroparesis. ${ }^{20}$ In humans with DGP, the switch from M2 to 
M1 macrophages in the gastric antrum is associated with decreased numbers of ICCs and gastric dysrhythmias. ${ }^{25}$ Thus, factors like macrophage switching and decreased ICC numbers, in addition to hyperglycemia, may be associated with the GMA dysfunctions in diabetic GP; and (d) sixteen percent of the GP subjects had normal $3 \mathrm{cpm}$ GMA. In these patients, pyloric dysfunction is a key factor in GP; and, pyloric therapies such as balloon dilation or injection of botulinum toxin A improve symptoms. ${ }^{22}$ Therefore, diabetic patients with $3 \mathrm{cpm}$ GMA in our cohort may not respond to insulin and may have confounded the CSII with CGM therapy results.

The strengths of this study are that it is (a) a multicenter study of well-characterized patients with diabetic gastroparesis with poorly controlled glycemia managed with CSII and CGM and (b) standard questionnaires, CSII with CGM treatment, and non-caloric and caloric test meals with standard tests of GMA were obtained. Weaknesses of the study include: (a) lack of a disease control group in regards to insulin therapy, dietary counseling, and concomitant medications during the 24 weeks of intensive CSII with CGM treatment, (b) lack of a healthy control group for symptoms and GMA in response to the CMST, (c) unknown numbers of subjects with glucose levels between $220 \mathrm{mg} / \mathrm{dL}$ and $270 \mathrm{mg} / \mathrm{dL}$ during the CMST and WLST, and (d) analyses of combined type 1 and type 2 diabetes mellitus subjects because of the small number of subjects in each group limits generalization of results for either group.

In summary, liquid caloric and non-caloric test meals immediately evoked significant increases in symptoms associated with gastroparesis. Almost $30 \%$ of patients ingested abnormally low volumes, suggesting poor gastric accommodation in a subset of patients with diabetic gastroparesis. Symptoms and $3 \mathrm{cpm}$ GMA increased after the CMST but not the WLST, indicating caloric meals are more potent stimulants of symptoms and $3 \mathrm{cpm}$ GMA. More aggressive or longer duration insulin therapy may be needed to affect symptoms and GMA in responses to provocative satiety test meals in patients with diabetic gastroparesis.

\section{ACKNOWLEDGMENTS}

The authors wish to thank Ms Stuart Pickett for her excellent secretarial assistance.

\section{CONFLICT OF INTEREST}

Dr Koch is a shareholder in 3CPM Company, Inc; other authors: no competing interests to disclose.

\section{AUTHORS CONTRIBUTIONS}

* See GpCRC Credit Roster. Kenneth L. Koch designed the study, contributed the enrollment of patients, critically revised the manuscript and important intellectual content, supervised the study. William L. Hasler designed the study, contributed the enrollment of patients, critically revised the manuscript and important intellectual content. Mark L. Van Natta analyzed and interpreted the data, critically revised the manuscript and important intellectual content. Madhusudan Grover critically revised the manuscript and important intellectual content. Pankaj J. Pasricha, William J. Snape, Thomas L. Abell, Linda Nguyen and Irene Sarosiek contributed the enrollment of patients, critically revised the manuscript and important intellectual content. Henry P. Parkman contributed the enrollment of patients, conceived the study and design, analyzed and interpreted the data and manuscript the writing. Richard W. McCallum conceived the study and design, contributed the enrollment of patients, critically revised the manuscript and important intellectual content. Gianrico Farrugia critically revised the manuscript and important intellectual content. James Tonascia designed the study, analyzed and interpreted the data, critically revised the manuscript and important intellectual content. Linda Lee and Laura Miriel analyzed and interpreted the data. Frank Hamilton critically revised the manuscript and important intellectual content.

\section{ORCID}

William L. Hasler (iD https://orcid.org/0000-0002-6158-2871

Madhusudan Grover (iD https://orcid.org/0000-0001-5092-0831

Henry P. Parkman (iD https://orcid.org/0000-0003-4904-4891

Thomas L. Abell ID https://orcid.org/0000-0002-3175-5161

Richard W. McCallum (iD https://orcid.org/0000-0002-6652-8049

Linda A. Nguyen (iD https://orcid.org/0000-0002-3498-6897

\section{REFERENCES}

1. Koch KL, Hasler WL, Yates KP, et al. Baseline features and differences in 48 weeks clinical outcomes in patients with gastroparesis and type 1 vs type 2 diabetes. Neurogastroenterol Mot. 2016;28:1001-1015

2. Karamanolis G, Caenepeel P, Arts J, Tack J. Determinants of symptom pattern in idiopathic severely delayed gastric emptying: gastric emptying rate or proximal stomach dysfunction? Gut. 2007;56:29-36

3. Pasricha PJ, Colvin R, Yates K, et al. Characteristics of patients with chronic unexplained nausea and vomiting and normal gastric emptying. Clin Gastroenterol Hepatol. 2011;9:567-576.

4. Tack J, Caenepeel P, Fischler B, Piessevaux H, Janssens J. Symptoms associated with hypersensitivity to gastric distention in functional dyspepsia. Gastroenterol. 2001;121:526-535.

5. Kindt S, Coulie B, Wajs E, Janssens J, Tack J. Reproducibility and symptomatic predictors of a slow nutrient drink test in health and in functional dyspepsia. Neurogastroenterol Motil. 2008;20: 320-329.

6. Kumar A, Attaluri A, Hashmi S, Schulze KS, Rao SS. Visceral hypersensitivity and impaired accommodation in refractory diabetic gastroparesis. Gastroenterol Motil. 2008;20:635-642.

7. Koch KL, Hong SP, Xu L. Reproducibility of gastric myoelectrical activity and the water load test in patients with dysmotility-like dyspepsia symptoms and in control subjects. J Clin Gastroenterol. 2000;31:125-129.

8. Parkman HP, Hallinan EK, Hasler WL, et al. satiety and postprandial fullness in gastroparesis correlate with gastroparesis severity, gastric emptying, and water load testing. Neurogastroenterol Motil. 2017;29(4):e12981. 
9. Calles-Escandon J, Koch KL, Hasler WL, et al. Glucose sensoraugmented subcutaneous insulin infusion in patients with diabetic gastroparesis: an open-label prospective study. PLoS ONE. 2018;13:e0194759.

10. Tougas G, Eaker EY, Abell TL, et al. Assessment of gastric emptying using a low fat meal: establishment of international control values. Am J Gastroenterol. 2000;95:1456-1462.

11. Koch KL. Electrogastrography for Evaluation of Patients with Suspected Gastroparesis. In: Parkman H, McCallum R,eds. Gastroparesis: Pathophysiology, Presentation, Diagnosis and Treatment. New York, NY: Springer;2011:153-161.

12. SAS Institute Inc. SAS version 9.4, SAS Institute Inc., Cary, NC.

13. StataCorp. Stata Statistical Software: Release 15. College Station, TX: StataCorp LLC; 2017.

14. Jones MP, Hoffman S, Shah D, Patek K, Ebert CC. The water load test: observations from healthy controls and patients with functional dyspepsia. Am J Physiol Gastrointest Liver Physiol. 2003;284(6):G896-904.

15. Grover M, Bernard CE, Pasricha PJ, et al. Clinical-histological associations in gastroparesis: results from the gastroparesis clinical research consortium. Neurogastroenterol Motil. 2012;24:5319.

16. O'Grady G, Angeli T, Du P, et al. Abnormal initiation and conduction of slow-wave activity in gastroparesis, defined by high-resolution electrical mapping. Gastroenterol. 2012;143:589-598.

17. Ladabaum U, Koshy SS, Woods ML, Hooper FG, OwYang C, Hasler WL. Differential symptomatic and electrogastrography effects of distal and proximal gastric stimulation. Am J Physiol. 1999;275:G418-G424.

18. Coleski R, Hasler WL. Coupling and propagation of normal and dysrhythmic gastric slow waves during acute hyperglycemia in healthy humans. Neurogastroenterol Motil. 2009;21:492-499.

19. Jebbink RJ, Samsom M, Bruijs PP, et al. Hyperglycemia induces abnormalities of gastric myoelectrical activity in patients with type 1 diabetes mellitus. Gastroenterol. 1994;107:1390-1397.
20. Faussone-Pellegrini MS, Grover M, Pasricha PJ, et al. Ultrastructural differences between diabetic and idiopathic gastroparesis. J Cell Mol Med. 2012;16:1573-1581.

21. Brzana RJ, Koch KL, Bingaman S. Gastric myoelectrical activity in patients with gastric outlet obstruction and idiopathic gastroparesis. Am J Gastroenterol. 1998;93:1803-1809.

22. Wellington J, Scott B, Kundu S, Stuart P, Koch KL. Effect of endoscopic pyloric therapies for patients with nausea and vomiting and functional obstructive gastroparesis. Auton Neurosci. 2017;202:56-61.

23. Reddy S, Ramsubeik K, Vega KJ, Federico J, Palacio C. Do HbA1c levels correlate with delayed gastric emptying in diabetic patients? J Neurogastroenterol Motil. 2010;16:414-417.

24. Lydon A, Murray C, Cooke T, Duggan PF, O'Halloran D, Shorten GD. Evaluation of standard haemodynamic tests of autonomic function and $\mathrm{HbA} 1 \mathrm{c}$ as predictors of delayed gastric emptying in patients with type 1 diabetes mellitus. Eur J Anaesthesiol. 2000;17:99-104.

25. Cipriani G, Gibbons SJ, Miller KE, et al. Change in populations of macrophages promotes development of delayed gastric emptying in mice. Gastroenterol. 2018;154:2122-2136.

How to cite this article: Koch KL, Hasler WL, Van Natta M, et al. Satiety testing in diabetic gastroparesis: Effects of insulin pump therapy with continuous glucose monitoring on upper gastrointestinal symptoms and gastric myoelectrical activity. Neurogastroenterol Motil. 2020;32:e13720. https://doi. org/10.1111/nmo.13720 